\title{
Características y percepciones sobre el uso de las plataformas de redes sociales y dispositivos tecnológicos por parte de los adolescentes
}

Sare sozialen plataformen eta gailu teknologikoen erabileraren ezaugarriak eta pertzepzioak nerabetan

Characteristics and perceptions about the use of social media platforms and technological devices by adolescents

Antonio García-Jiménez`, María Cruz López-de-Ayala López, Manuel Montes-Vozmediano

Universidad Rey Juan Carlos

RESUMEN: El crecimiento exponencial del acceso a dispositivos móviles está fomentando un uso ubicuo y más intensivo de ciertos servicios y aplicaciones online. Este artículo aborda las características de los usos que los menores realizan tanto de los dispositivos de acceso a las redes sociales como de las plataformas que las contienen. Mediante una encuesta aplicada a una muestra representativa de adolescentes de la Comunidad de Madrid matriculados en la ESO ( $\mathrm{N}=524)$, se estudia la edad de propiedad del primer smartphone, la frecuencia de uso de las plataformas de redes sociales y de WhatsApp, las actividades que realizan y su frecuencia, y finalmente sus valoraciones respecto a las redes sociales. Algunos de los hallazgos tienen que ver con la confirmación de la tendencia de las edades para la adquisición del propio smartphone (media de 11 años). Edad que también se muestra como el referente para el acceso a las redes sociales. Junto al acceso multipantalla, también se observa un consumo diario superior de Whatsapp en relación con el de las plataformas de redes sociales. Por otra parte, las necesidades de socialización y la búsqueda de entretenimiento orientan el uso online de la adolescencia. Finalmente, los adolescentes eligen mayoritariamente Instagram para realizar la mayoría de sus actividades en redes sociales; y, a continuación, YouTube, en el caso de contenidos audiovisuales.

PALABRAS CLAVE: adolescentes, menores, redes sociales, usos, plataformas.

ABSTRACT: The exponential growth of access to mobile devices is encouraging a ubiquitous and more intensive use of certain online services and applications. This article addresses the characteristics of the uses that minors make of both social networking access devices and the platforms that contain them. Through a survey applied to a representative sample of teenagers in the Community of Madrid enrolled in ESO $(N=524)$, the age of ownership of the first smartphone, the frequency of use of social networking platforms and WhatsApp, the activities they perform and their frequency, and finally their assessments regarding social networks are studied. Some of the findings are related to the confirmation of the trend of ages for the acquisition of the smartphone itself (11 years old on average). Age is also shown as the reference for access to social networks. Along with multi-screen access, a higher daily consumption of Whatsapp is also observed in relation to the others social network platforms. On the other hand, socialization needs and the search for entertainment are the key factors in online use of adolescents. Finally, teenagers mostly choose Instagram to carry out most of their activities on social networks; and then YouTube, in the case of audiovisual content. KEYWORDS: adolescents, minors, social networks, uses, platforms.

\footnotetext{
* Correspondencia a / Corresponding author: Antonio García-Jiménez. Universidad Rey Juan Carlos. Facultad de Ciencias de la Comunicación. Camino del Molino, s/n (29843 Fuenlabrada-Madrid) - antonio.garcia@urjc.es - https://orcid.org/0000-0002-8423-9486

Cómo citar / How to cite: García-Jiménez, Antonio; López-de-Ayala López, María Cruz; Montes-Vozmediano, Manuel (2020). "Características y percepciones sobre el uso de las plataformas de redes sociales y dispositivos tecnológicos por parte de los adolescentes", Zer, 25(48), 269-286. (https://doi.org/10.1387/zer.21556).
}

Recibido: 17 marzo, 2020; aceptado: 17 abril, 2020.

ISSN 1137-1102 - eISSN 1989-631X / (c) 2020 UPV/EHU

(C) (i) Esta obra está bajo una licencia 


\section{Introducción}

En la actualidad, los y las adolescentes constituyen uno de los principales colectivos de mayor uso y consumo de tecnología y medios sociales. La investigación pone de manifiesto que el inicio en el uso de estas herramientas se va produciendo paulatinamente a edades cada vez más tempranas (Garmendia, Jiménez, Casado y Mascheroni, 2016; Livingstone, Haddon, Görzig y Ólafsson, 2011). El informe Net Children Go Mobile en España de 2016 situaba los 7 años como la edad media de inicio en el uso de internet (Garmendia et al., 2016). En la adolescencia, el uso de internet constituye un hábito generalizado, el 92,3\% según el INE (2019) y el 97,7\% según datos de una macroencuesta realizada con adolescentes españoles (GolpeFerreiro, Gómez-Salgado, Harris, Braña y Rial, 2017).

Los dispositivos portátiles (teléfono inteligente, tableta y ordenador portátil) han cogido protagonismo en los últimos años y son los más utilizados para acceder a internet en la adolescencia (Pastor Ruiz, Martín Nieto y Montes Vozmediano, 2019). Datos del Instituto Nacional de Estadística (INE, 2019) corroboran que el $66 \%$ de los adolescentes españoles entre 10 y 15 años disponen de su propio teléfono móvil y el $89,7 \%$ posee un ordenador personal. Entre todos los dispositivos destaca la notable expansión del uso del smartphone en la adolescencia (Garmendia et al., 2016; Viñals, 2016). Según datos del Pew Research Center (PRC) en Estados Unidos, el 95\% de los adolescentes posee uno en propiedad (Anderson y Jiang, 2018) y el 91,7\% entre los adolescentes españoles (Golpe-Ferreiro et al., 2017). No existe un dato claro de la edad media de posesión del primer smartphone para uso exclusivo y personal, aunque algunas organizaciones no gubernamentales sitúan la edad media sobre los 12-13 años y parece que la tendencia es a adelantarse progresivamente. El estudio más reciente realizado con adolescentes españoles sitúa los 11,12 años como la edad media de acceso al primer móvil, aunque en el mismo no especifican si se trata de un teléfono inteligente (Golpe-Ferreira et al., 2017).

La extensión del uso de los dispositivos portátiles ha favorecido un aumento en la intensidad de uso de internet por parte de los adolescentes. Así, por ejemplo, el informe de PRC en USA indicaba que el 45\% de los adolescentes afirmaba estar utilizando internet constantemente y pronosticaba que este dato iría en aumento en los próximos años (Anderson y Jiang, 2018). Estudios cualitativos realizados en nuestro país confirman esta tendencia también entre los adolescentes españoles (Cuesta y Gaspar, 2013; Pastor Ruiz et al., 2019). Otros trabajos informan que el 73,7\% de los adolescentes se conecta a internet a diario y el $77 \%$ le dedica más de una hora al día, siendo la franja de conexión predilecta entre las 16 y las 21 horas (Golpe-Ferreiro et al., 2017). La realización de múltiples tareas, como estudiar o escuchar música, a la par que están conectados a internet viendo otras cosas o contactando con amigos forma parte de la práctica habitual de los y las adolescentes (Navarro, García, González, Contreras y Massana, 2012; Sendín, Gaona y García, 2014), incluso con fre- 
cuencia llevan a cabo un acceso simultáneo de varios dispositivos y aplicaciones (Fernández-Planells y Figueras, 2012).

Muchos jóvenes incluso informan pasarlo mal si no disponen de acceso a internet o no llevan el móvil encima, y admiten tener dificultades para autorregular su uso cuando tienen que estudiar o para dormir (Pastor Ruiz et al., 2019). Algunas organizaciones no gubernamentales están realizando llamamientos alertando del mal uso del smartphone en la etapa adolescente. Así, por ejemplo, el Instituto Psicológico Desconecta, como resultado de una encuesta realizada con personas entre 10 y 60 años, expone que algo más del 40\% de los adolescentes entre 10 y 14 años mantienen un contacto activo con el móvil (lo desbloquean y encienden la pantalla) cada 15-30 minutos, lo que supone entre 50 y 100 veces al día. Esta costumbre aumenta considerablemente entre los 15 y 17 años, edad en la que un porcentaje similar tiende a mantener un contacto activo con el dispositivo en una frecuencia entre 5 y 7 minutos (Masip y Balagué, 2015). En consonancia con este dato empiezan a proliferar estudios sobre el FOMO (Fear of Missing Out), que consiste en una especie de temor o miedo a perderse algo por no estar conectado a la red (Burglass, Binder, Betts y Underwood, 2017).

Las redes sociales, la mensajería instantánea y las aplicaciones para escuchar música constituyen las herramientas online más frecuentemente utilizadas por los adolescentes españoles (Golpe-Ferreiro et al., 2017; Reolid-Martínez et al., 2016). Los datos indican que el 93,5\% están registrados en alguna red social y el 90,7\% de los adolescentes utilizan el WhatsApp como herramienta de mensajería instantánea. Las redes sociales predilectas para los adolescentes españoles son Instagram, Twitter y Facebook (Golpe-Ferreiro et al., 2017), mientras que en otros países como USA triunfan YouTube, Instagram y Snapchat (Anderson y Jiang, 2018). Respecto a las actividades más frecuentes entre los adolescentes en el uso de las redes encontramos: relacionarse con amigos y conocidos, estudiar, descargar materiales, escuchar música, ver películas y series, los juegos online, subir información y pasar el tiempo/ocio (Golpe-Ferreiro et al., 2017), sin evidenciarse en estos hábitos unas diferencias reseñables en cuestión de género, por lo que las pautas de comportamiento en chicos y chicas siguen aproximándose, una tendencia constatada en estudios previos (Garmendia, Garitaonandia, Martínez y Casado, 2011).

No obstante, sí es factible encontrar aún ciertas pautas con una mayor prevalencia según el género, manifestándose en los chicos la preferencia por los juegos online (Ak, Koruklu y Yilmaz, 2013) y en las chicas, un porcentaje mayor de uso de algunas redes consideradas de mayor contenido icónico o visual como Instagram o Pinterest a las que dan un uso más social (Lenhart, Smith y Anderson, 2015; Golpe Ferreiro et al., 2017). Así pues, se observa cierta tendencia a llevar a cabo un uso más social de los medios en el caso de las chicas y un uso más lúdico en el caso de los chicos (Fernández-Montalvo, Peñalva e Irazabal, 2015). 
Cuando se trata de formarse o localizar información, los adolescentes optan por plataformas como YouTube (Pérez-Torres, Pastor-Ruiz y Abarrou-Ben-Boubaker, 2018) en las que localizan audiovisuales formativos con los temas de su interés y presentados con un enfoque positivo (García Jiménez y Montes Vozmediano, 2020).

La realización de descargas es otra de las motivaciones que tienen los adolescentes para conectarse a internet, desde las tradicionales descargas de juegos, programas o películas (Martínez-Pastor, Sendín-Gutiérrez y García-Jiménez, 2013) a las que se incorporan las descargas de canciones y otros contenidos audiovisuales (vídeos, fotos) con fines, fundamentalmente, lúdicos y de entretenimiento (Espín Álvarez y Freire Muñoz, 2019).

En lo que respecta a sus preferencias sobre el contenido sonoro que escuchan, para los jóvenes, la música se antepone a otras modalidades como los espacios de palabra (de radio o podcast). En sus elecciones musicales prevalecen los criterios personales del adolescente, pues no son tan influenciables por las sugerencias de sus amigos o familiares, para su consumo tienden a aislarse del entorno mediante auriculares y su plataforma preferencial es Spotify (Pedrero-Esteban, Barrios-Rubio y MedinaÁvila, 2019). Estos mismos investigadores han constatado que los jóvenes también recurren a la plataforma YouTube para escuchar música - les permite, además, un consumo en formato audiovisual - y lo correlacionan con el uso del smartphone, un dispositivo que demanda explícitamente la atención visual.

En lo que respecta al consumo de contenido audiovisual, internet relega a la televisión, ya que facilita la elección personalizada del qué, cuándo, dónde y cómo se visualiza el contenido. Los jóvenes se interesan por las series de ficción, las películas o los programas de entretenimiento (García Jiménez, Tur-Viñes y Pastor Ruiz, 2018).

El afán de los adolescentes de ser actores protagonistas en la red, para lo cual se decantan por plataformas como YouTube (Aguaded y Sánchez, 2013) o redes sociales como Instagram, les lleva a realizar acciones desaconsejables como publicar imágenes o vídeos realizados en espacios privados (Montes-Vozmediano, GarcíaJiménez y Menor-Sendra, 2018) al imitar las pautas de sus referentes en el ámbito online, si bien estos suelen ser youtubers o instagrames adultos, cuyos canales constituyen una forma de negocio. Puesto que la generación de contenido es uno de los usos que se acrecienta entre los adolescentes, deben conocer las posibles derivaciones o consecuencias de su actividad, tanto para su propia protección como para aconsejar a otros iguales en situaciones similares (Jiménez Iglesias, Garmendia y Casado del Río, 2015).

Las valoraciones que hacen los adolescentes de los medios sociales son variadas. La mayoría de ellos considera que los medios sociales poseen tanto efectos positivos 
como negativos sobre sus vidas (45\%), el $31 \%$ considera que solo posee beneficios mientras que el $24 \%$ percibe que son perniciosos. Entre los beneficios reportados con mayor frecuencia encontramos la conectividad, la facilidad de estar en contacto con amigos y familiares, y también el acceso a la información de todo tipo. Entre los perjuicios más nombrados se halla el acoso (Bullying), la información falsa que se difunde en la red con facilidad, la distorsión de la realidad y el exceso de tiempo invertido (Anderson y Jiang, 2018). En un estudio sobre las actitudes de los adolescentes españoles hacia internet y las redes sociales, Rial, Gómez, Braña y Varela (2014) encontraron que la mayoría de ellos perciben que la gente de su edad realiza un uso excesivo e inapropiado de internet, mientras que ellos mismos se perciben con bajo riesgo de dependencia de la red. En términos generales, entienden que internet es un lugar seguro, creen que utilizan medidas suficientes para protegerse en la red y no consideran que ellos mismos lleven a cabo un uso inapropiado (Ballesta, Lozano, Cerezo y Soriano, 2015).

Los objetivos del presente estudio, que se configuran como una parte de una investigación de mayor dimensión, giran alrededor de las siguientes cuestiones de investigación:

P1. Nos preguntamos por la edad a la que se tuvo en propiedad un smartphone por parte de los menores ( $\dot{\mathrm{A}}$ qué edad tuviste tu primer smartphone con acceso a internet?).

P2. El segundo punto de análisis tiene que ver con los dispositivos de acceso a internet, en general, y a las redes sociales en particular, ya sean el teléfono móvil, el ordenador personal o la tableta (¿Desde qué tipo de dispositivo sueles acceder a medios o redes sociales sin tener en cuenta WhatsApp?).

P3. También es relevante conocer la frecuencia de uso de las redes sociales y de WhatsApp (¿Me puedes decir con qué frecuencia usas rede sociales (Facebook, Tuenti, Instagram, Twitter...? ¿Y WhatsApp?).

$\mathrm{P} 4$. El siguiente punto es el referido a la frecuencia con la que se realizan determinadas actividades en internet por parte de los encuestados. En este punto, interesan acciones como la descarga y escucha de música en las plataformas correspondientes, hacer recomendaciones y leer memes, jugar online o descargar y ver películas y series (A continuación te mostramos una serie de actividades que puedes hacer en Internet. Marca con una X con qué frecuencias lo haces tú para cada una de ellas).

P5. También interesan las valoraciones hacia los medios sociales. Son pertinentes cuestiones como la percepción sobre el tiempo que se les dedica o bien el impacto que tienen en su vida cotidiana, su relación con el mundo offline, o la capacidad de las mismas para la integración en el grupo (Señala tu grado de acuerdo o desacuerdo con cada una de las siguientes afirmaciones). 
P6. Finalmente, se revisan las actividades realizadas por los menores en las redes sociales, sin incluir WhatsApp. Las redes sociales sobre las que se pregunta son Instagram, YouTube, Snapchat, Twitter y Facebook (De las diferentes actividades por los que puedes conectarte a redes y medios sociales (sin tener en cuenta WhatsApp), ¿con qué frecuencia las realizas tú? ¿y en qué redes sociales sueles hacerlo?).

\section{Metodología}

Este estudio se ha realizado a partir de los datos recopilados en una encuesta aplicada a una muestra estadísticamente representativa $(n=524)$ de alumnos matriculados en Educación Secundaria Obligatoria $\left(1 .^{\circ}\right.$ a $4 .^{\circ}$ de la ESO) de la Comunidad de Madrid, durante el curso académico 2019-2020.

El diseño de la muestra siguió un muestro polietápico estratificado por conglomerados. La estratificación se realizó en función de la titularidad del centro (público, privado o concertado) y discriminando entre Madrid capital y el resto de municipios de la Comunidad de Madrid. Los conglomerados correspondían a los centros educativos de ESO en un primer nivel. En total se seleccionaron 16 centros educativos. Una vez que los centros aceptaron su participación en la encuesta, se seleccionaron las unidades de análisis de la segunda etapa que estaban constituidas por las aulas. Todos los alumnos del aula que contaban y presentaron la correspondiente autorización previa participaron en la encuesta. El análisis de error se fijó en $\pm 4,37 \%$ para los datos globales bajo el supuesto de $\mathrm{p}=\mathrm{q}=0,5$ con un nivel de confianza del $95 \%$, y bajo el supuesto de un muestreo aleatorio simple.

El trabajo de campo fue realizado entre el 24 de octubre al 19 de diciembre de 2019. El acceso a la información se realizó por el sistema C.A.P.I. Las entrevistas se realizaron de forma personal en los colegios aplicando cuotas de sexo, ámbito y curso. Se recogieron las correspondientes autorizaciones de los colegios y los consentimientos pertinentes. El tiempo de cumplimentación de la encuesta fue de alrededor de 45 minutos. Los ítems propuestos fueron de elaboración propia.

Los datos recogidos fueron ponderados manteniéndose las proporciones de la distribución real de la población según la distribución por tipo de municipio (Madrid y resto de municipios de la Comunidad de Madrid), tipo de centro, nivel educativo y sexo de los encuestados (según los datos de la Oficina de Estadística y Consejería de Educación de la Comunidad de Madrid). Para el análisis de los datos, y mediante el programa SPSS, se han realizado tablas de contingencia y las diferencias significativas se han situado en el nivel de validez estadística de $\chi^{2}<0.05$. 


\section{Resultados}

\subsection{EDAD DE ACCESO}

La mayor parte de los encuestados (34\%) afirma que fue a los 12 años cuando tuvo en propiedad su primer smartphone. A partir de ahí, y en sentido decreciente se observa un menor porcentaje. Así, a los 11 años, fue el 26,4\%, y la edad de 10 años, aproximadamente el $18 \%$. Se puede destacar que antes de los 10 años casi el $12 \%$ declara que tuvo su primer teléfono inteligente. Finalmente, el porcentaje más reducido es el que se sitúa entre los 13 y los 16 años. En consecuencia, se puede considerar que la edad media es de 11 años (moda a los 12 y desviación típica de 1,6 años).

En lo que se refiere a la edad de inicio en el uso/acceso a los medios sociales, casi el 49\% afirma que comenzó en este mundo entre los 11 a 12 años. A continuación, la edad con un mayor porcentaje de respuestas es la de mayor de 12 años, con un $26,2 \%$. En tercer lugar, de 9 a 10 , con casi un 16\%. Sobresale también el porcentaje de menores que se inicia en el uso/consumo de redes sociales antes de los 8 años, un total del $6,3 \%$.

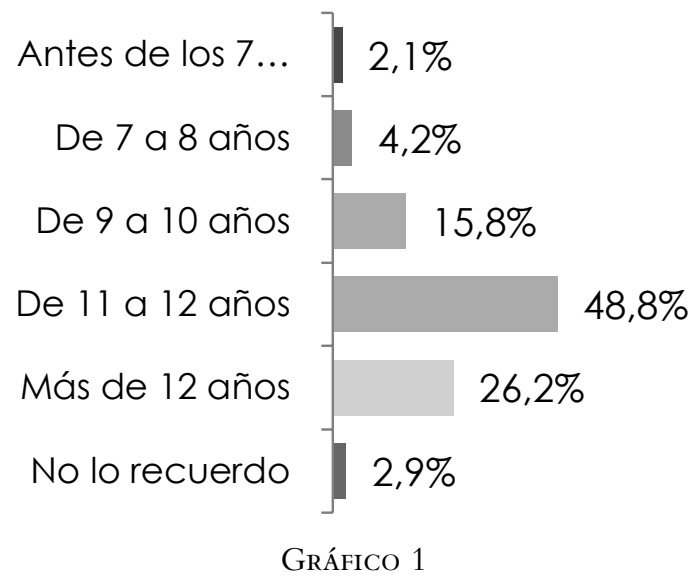

Edad de comienzo de uso de las redes sociales

Dispositivos de acceso/uso

El segundo punto de análisis tiene que ver con los dispositivos de acceso. El dispositivo más utilizado para conectarse y acceder a las redes sociales es el teléfono móvil $(88,5 \%)$ a gran distancia del ordenador personal $(42,2 \%)$ o la tableta, dispositivos que se colocan en la segunda posición del ranking con datos de utilización bastante parecidos. 
En cuanto a las diferencias significativas entre segmentos, destacan que los chicos utilizan significativamente más otros dispositivos a los analizados (teléfonos inteligentes, ordenador propio o de sobremesa, tableta, Smart TV o bien ordenador de otros miembros de la familia). Estos «otros dispositivos» podrían hacer referencia a las videoconsolas, dispositivo que parece que significativamente son más utilizados por los varones. Otra diferencia significativa de relevancia es el hecho de que los alumnos del primer curso de secundaria utilizan más las tabletas en comparación con el resto de cursos académicos.

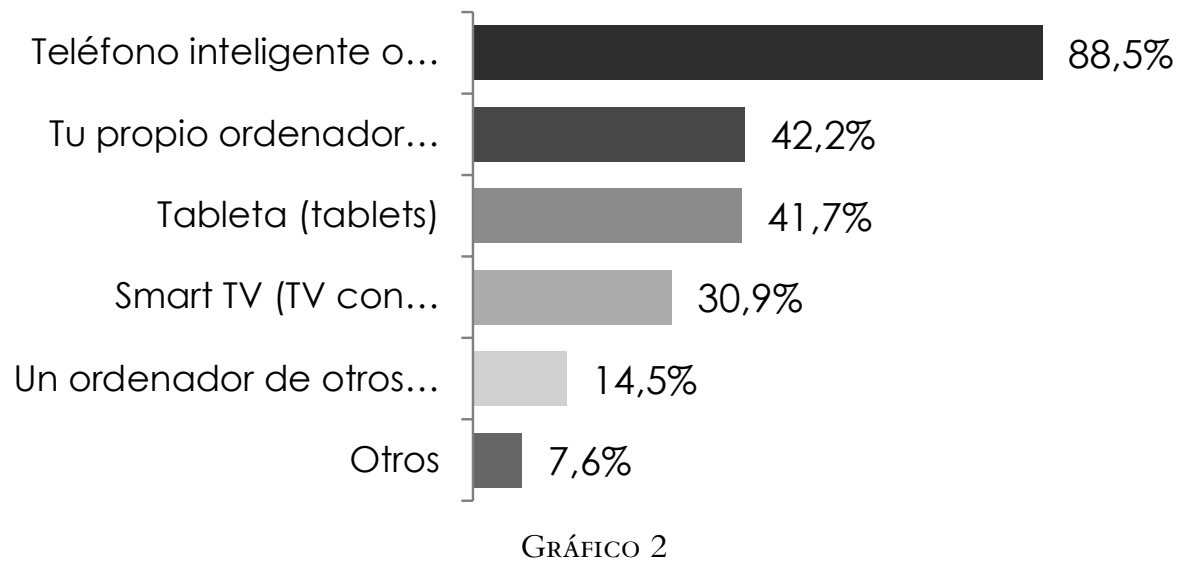

Dispositivo desde el que se suele acceder a medios o redes sociales sin tener en cuenta WhatsApp

Frecuencia de uso de redes sociales y aplicaciones de mensajería

El $28,8 \%$ de los encuestados afirma que accede a las redes sociales continuamente. A su vez, el 24,5\% declara que lo hace varias veces al día y, por su parte, un rato todos los días el $21 \%$. Si sumamos estos tres tipos de respuestas, se advierte que más del $74 \%$ de los menores usa las redes sociales diariamente, en diferentes niveles de consumo. Por otro lado, también destaca la cifra baja de menores que lo usan 3-4 días a la semana $(5,2 \%)$, un día a la semana (3\%) o con menos frecuencia $(5,4 \%)$. A su vez, el 12,3\% declara que no consume redes sociales.

En el caso de WhatsApp, el uso diario es de un 83,3\%, que es mayor que el uso comparado con el de las plataformas de redes sociales. Aquí, el dato referido a los menores que acceden continuamente es de un $28,8 \%$ (el mismo que el porcentaje que señala que consulta de modo continuo las redes sociales). No obstante, la categoría que ofrece más frecuencia es la referida a varias veces al día, con un 29,5\%; y un rato todos los días el 25\%. Finalmente, los que usan 3-4 días a la semana representan el 7,7\% y aquellos que dicen que no lo usan el 5,3\%. 


\subsection{FreCUENCIA DE ACTIVIDAdes EN INTERNET}

La actividad realizada con mayor frecuencia por los adolescentes en internet es la referida a la descarga y a la escucha de música en las plataformas dedicadas a ello $(65,4 \%)$. A gran distancia, en un segundo escalón, se encuentran actividades más centradas en el ocio y la diversión como pueden ser la de observar las recomendaciones y memes $(38,5 \%)$, jugar online $(35,7 \%)$ o descargar y ver películas y series $(35,2 \%)$. Ya en menor medida la consulta de información de actualidad, la de acceso a páginas de adultos, y las páginas de compra o venta online.

\section{TABLA 1}

Frecuencia de actividades en Internet.

Porcentajes referidos a la suma de las opciones continuamente o con mucha frecuencia

\begin{tabular}{ll}
\hline Descargar o escuchar música (Spotify, iTunes) & $65,4 \%$ \\
\hline Mirar las recomendaciones y memes que me llegan & $38,5 \%$ \\
\hline Jugar a juegos online & $35,7 \%$ \\
\hline Descargar o ver películas o series, televisión online & $35,2 \%$ \\
\hline Consultar información de actualidad online (noticias) & $20,7 \%$ \\
\hline Participar en mundos virtuales (Minecraft, Roblox, ...) & $18,8 \%$ \\
\hline Acceder a páginas de sexo/de adultos & $10,4 \%$ \\
\hline Hacer compras o ventas online (Wallapop o similar) & $9,5 \%$ \\
\hline Buscar información sobre relaciones sexuales & $7,0 \%$ \\
\hline Acceder a páginas de búsqueda de pareja & $5,4 \%$ \\
\hline Buscar información sobre cómo consumir o dónde conseguir drogas & $5,1 \%$ \\
\hline Acceder a páginas de apuestas deportivas o casino online & $4,8 \%$ \\
\hline
\end{tabular}

\subsection{OpINIÓN SOBRE LAS REDES SOCIALES}

En general, las valoraciones hacia las redes sociales de los adolescentes son divergentes. Por un lado, hay categorías de carácter positivo que tienen detrás porcentajes relevantes de los encuestados. Pero por otro, y aunque en general las afirmaciones negativas no muestran un seguimiento muy alto, el 51,6\% de los menores admite que las redes sociales les hacen perder mucho tiempo. Y se trata de la percepción con mayor seguimiento. A pesar de admitir esto, los encuestados no piensan que aíslen de la vida real, y entienden que a través de estas pueden expresar sus opiniones libremente (45\%). A continuación, destaca la categoría refe- 
rida a la sensación de que les permite estar más integrado en su grupo de amigos $(31,8 \%)$.

Frente a esto, hay un porcentaje importante de menores que entiende que el mundo de las redes sociales o bien te aísla de la vida fuera del marco digital (29,5\%) o bien se sienten presionados para contestar inmediatamente a los mensajes que reciben, ya sea por medios de las plataformas de redes sociales o mediante el WhatsApp $(27,5 \%)$. A su vez, el 23,1\% afirma que, a menudo, ha tenido problemas de malos entendidos, lo que ha podido llevar a perder un amigo. Vinculados más a riesgos, el $22,3 \%$ afirma que se pone nervioso pensando que se puede estar perdiendo alguna cosa interesante o incluso llegan a sentirse controlados (20\%) o sentirse presionados para publicar o dar un «like» $(16,4 \%)$.

\section{TABLA 2}

Opinión sobre las redes sociales.

Porcentajes referidos a la suma de las opciones totalmente de acuerdo y de acuerdo en relación a los ítems

\begin{tabular}{|c|c|}
\hline Las redes sociales me hacen perder mucho tiempo & $51,6 \%$ \\
\hline A través de redes sociales, puedo expresar mis opiniones & $45,0 \%$ \\
\hline Las redes sociales me hacen sentir más integrado en mi grupo de amigos & $31,8 \%$ \\
\hline Las redes sociales te aíslan de la vida real & $29,5 \%$ \\
\hline $\begin{array}{l}\text { A menudo me siento presionado/a a contestar inmediatamente a los mensajes que me } \\
\text { llegan por redes sociales o WhatsApp }\end{array}$ & $27,5 \%$ \\
\hline $\begin{array}{l}\text { A menudo he tenido problemas de malos entendidos en redes sociales o WhatsApp, } \\
\text { hasta el punto de perder un amigo/a }\end{array}$ & $23,1 \%$ \\
\hline $\begin{array}{l}\text { Cuando no tengo acceso a las redes sociales y/o WhatsApp, me pongo nervioso/a } \\
\text { pensando que me puedo estar perdiendo algo }\end{array}$ & $22,3 \%$ \\
\hline A veces me siento totalmente controlado en las redes sociales & $20,0 \%$ \\
\hline $\begin{array}{l}\text { A menudo me siento presionado/a a publicar contenidos en redes sociales que obten- } \\
\text { gan muchos likes y me gusta }\end{array}$ & $16,4 \%$ \\
\hline
\end{tabular}

Si profundizamos más en esta cuestión, se puede observar la incidencia del curso en el que se está matriculado en la valoración de las redes sociales. Seleccionadas las cuatro opiniones con mayor porcentaje de acuerdo, se advierten diferencias estadísticamente significativas en todas ellas. En cuanto a la consideración de que las redes sociales te "hacen perder mucho el tiempo", esta aumenta con el curso: un $42,2 \%$ lo afirman los estudiantes en $1 .^{\circ}$ de la ESO (si sumamos los que están «bastante de acuerdo» y los que afirman estar «totalmente de acuerdo»), llegando al $60,2 \%$ en $4 .^{\circ}$ curso. 
La segunda de las opiniones más recurrentes estaba relacionada con la capacidad de expresar las propias opiniones a través de las redes. Son más los que no están de acuerdo (o bien "nada de acuerdo» o bien "un poco de acuerdo») a principio de la secundaria que el último curso: el $47,1 \%$ en $1 .^{\circ}$ de la ESO, el $21,4 \%$ en $4 .^{\circ}$.

\section{TABLA 3}

Opinión sobre las redes sociales según el curso. Porcentajes verticales. Diferencias significativas a un $\mathrm{p}$ valor de $\chi^{2}<0,05$.

Los valores destacados son significativamente diferentes para $\mathrm{p}<0.05$ en el test de igual bilateral para proporciones de columnas

\begin{tabular}{|c|c|c|c|c|}
\hline & 1. ${ }^{\circ} \mathrm{ESO}$ & 2. ${ }^{\circ} \mathrm{ESO}$ & 3. ${ }^{\circ} \mathrm{ESO}$ & $4 .^{\circ} \mathrm{ESO}$ \\
\hline \multicolumn{5}{|c|}{ Las redes sociales me hacen perder mucho tiempo } \\
\hline Nada de acuerdo & 22,7 & 12,5 & 8,0 & 7,7 \\
\hline Poco de acuerdo & 9,0 & 12,7 & 12,6 & 11,5 \\
\hline $\mathrm{Ni}$ acuerdo/ni desacuerdo & 26,1 & 21,3 & 27,3 & 20,6 \\
\hline Bastante de acuerdo & 20,3 & 24,2 & 31,4 & 39,4 \\
\hline Totalmente de acuerdo & 21,9 & 29,3 & 20,7 & 20,8 \\
\hline \multicolumn{5}{|c|}{ A través de las redes sociales, puedo expresar mis opiniones } \\
\hline Nada de acuerdo & 20,7 & 13,7 & 10,8 & 7,6 \\
\hline Poco de acuerdo & 26,4 & 7,9 & 12,5 & 13,8 \\
\hline $\mathrm{Ni}$ acuerdo/ni desacuerdo & 23,7 & 28,7 & 28,3 & 23,8 \\
\hline Bastante de acuerdo & 16,0 & 28,1 & 29,0 & 38,1 \\
\hline Totalmente de acuerdo & 13,2 & 21,6 & 19,4 & 16,7 \\
\hline \multicolumn{5}{|c|}{ Las redes sociales me hacen sentir más integrado en mi grupo de amigos } \\
\hline Nada de acuerdo & 38,1 & 29,9 & 19,6 & 16,3 \\
\hline Poco de acuerdo & 16,5 & 19,9 & 15,3 & 22,5 \\
\hline $\mathrm{Ni}$ acuerdo/ni desacuerdo & 11,9 & 21,1 & 31,0 & 30,7 \\
\hline Bastante de acuerdo & 18,6 & 15,1 & 21,6 & 20,5 \\
\hline Totalmente de acuerdo & 14,9 & 14,0 & 12,5 & 10,0 \\
\hline \multicolumn{5}{|c|}{ Las redes sociales te aíslan de la vida real } \\
\hline Nada de acuerdo & 35,6 & 22,1 & 27,6 & 13,0 \\
\hline Poco de acuerdo & 15,6 & 11,8 & 12,7 & 25,8 \\
\hline $\mathrm{Ni}$ acuerdo/ni desacuerdo & 31,5 & 28,9 & 28,1 & 28,8 \\
\hline Bastante de acuerdo & 8,5 & 14,6 & 17,8 & 19,1 \\
\hline Totalmente de acuerdo & 8,8 & 22,6 & 13,7 & 13,3 \\
\hline
\end{tabular}

En cuando a la valoración de las redes sociales como herramientas que les permiten sentirse integrados dentro de su grupo de amigos, esta se mantiene estable a lo largo de los cuatro cursos. Las diferencias significativas se observan entre los que no están nada de acuerdo con esta afirmación: es el caso del 38,1\% de los alumnos de 
primero, si bien esta cifra se reduce al 16,3\% en cuarto de la ESO. A su vez, si se suman los resultados de las categorías «nada de acuerdo" y "poco de acuerdo", las diferencias entre los menores de primer y cuarto curso disminuyen.

Finalmente, en cuanto a la posibilidad de que las redes sociales aíslen de la «vida real», también se observa que los alumnos de cursos más bajos se muestran, en mayor proporción, «nada de acuerdo» con esta idea. Es lo que ocurre para el 35,6\% de los alumnos de $1 .^{\circ}$ de ESO, mientras que solo el $13 \%$ de los matriculados en $4 .^{\circ}$ de la ESO estarían «nada de acuerdo» con esta valoración. Estos datos también se reducen en el caso de sumar las categorías «nada de acuerdo» y «poco de acuerdo».

\subsection{Actividades en las Redes Sociales}

Lo primero que destaca en relación a las actividades realizadas en las redes sociales, las acciones que más llevan a cabo los adolescentes hacen referencia a hablar con amigos y/o familiares y a ver vídeos y música.

Por su parte, la red social más utilizada, en general para todas las actividades, es a cierta distancia Instagram, que sobresale en las diferentes actividades: publicar contenidos personales $(93,3 \%)$; realizar actualizaciones sobre lo que hago o dónde estoy $(91,1 \%)$; ver vídeos o fotos de amigos y familiares $(86,4 \%)$, ver vídeos o fotos de desconocidos o perfiles abiertos $(91,6 \%)$; hablar con amigos $(86,2 \%)$ o hablar con familiares (45,5\%); y buscar información sobre famosos $(77,45 \%)$.

No obstante, YouTube sobresale en las categorías de ver vídeos y música (92,1\%); jugar online (donde destaca la categoría de otros con un 55\% y después YouTube con un 33,5\%); buscar contenidos sobre los hobbies $(72,1 \%)$; buscar contenido deportivo $(66,7 \%)$; buscar información sobre ocio $(58,8 \%)$; buscar series, películas o programas de televisión (70,3\%); y buscar información sobre salud/nutrición y bienestar (54\%).

A gran distancia ya aparecen, y por este orden, Snapchat, Twitter y Facebook. Estas dos últimas con un porcentaje medio bajo. Finalmente, hay que destacar la importancia creciente de otras redes y plataformas surgidas recientemente como Tik Tok o Twitch.

\section{Conclusiones}

El crecimiento exponencial del acceso a dispositivos móviles, y en particular del smartphone, está fomentando un uso ubicuo y más intensivo de ciertos servicios y aplicaciones online y modifica las formas de relación con los soportes y conte- 
nidos en formato audiovisual. En particular, los medios sociales se han convertido en un elemento central en la vida digital de adolescentes y jóvenes, convirtiéndose en auténticos espacios para desplegar todo tipo de actividades, con códigos propios de comportamiento y diferenciación de significados, y con tendencia a la convergencia. En este artículo, se ha buscado actualizar el conocimiento sobre el uso digital de los adolescentes en cuanto que usuarios muy activos, que los convierte, junto con su menor experiencia, en sujetos vulnerables a los riesgos online. En particular, se prestó especial atención al uso de las redes sociales y el significado que los adolescentes les otorgan, en cuanto espacios que concentran un mayor riesgo relacionado con su capacidad para crear y compartir contenidos entre pares y sumando a los efectos de persuasión de los medios, la influencia interpersonal (Fogg, 2008).

En primer lugar, se confirma que la norma para que los adolescentes adquieran su propio smartphone se sitúa en los doce años, siendo la edad media un poco más temprana: los once años. Estos datos coinciden con los encontrados por GolpeFerreiro et al. (2017) y corroboran que la edad de entrada a la adolescencia se sitúa socialmente en los doce años, momento en el que los padres facilitan la propiedad de este dispositivo a sus hijos como rito de tránsito hacia una etapa marcada por mayor autonomía personal. También sugiere que esta edad se adelanta en algunas ocasiones, ya sea por circunstancias familiares o personales particulares que obligan a supervisar a distancia a sus hijos antes de lo que incluso muchos padres desean, o bien porque algunos padres consideran que es mejor facilitárselo más jóvenes para poder modelar y controlar su uso cuando aún pueden influir en ellos (López-de-Ayala, MartínezPastor y Catalina-García, 2019).

Los once o doce años también se convierten en el referente para el acceso a las redes sociales, aunque en este caso es más habitual que se retrase, con casi un tercio de adolescentes que accedieron a una edad superior y solo un $22 \%$ que lo usó a una edad más temprana. A partir de esa edad, el uso de estas herramientas es muy intensivo, con tres cuartas partes de los adolescentes que las utilizan diariamente y más de una cuarta parte que lo hacen continuamente. Unicamente un $12 \%$ de encuestados señalan que no las usa.

Por otra parte, nuestros datos no coinciden con los resultados de GolpeFerreiro et al. (2017) que apuntan a una mayor penetración de las plataformas de redes sociales que del WhatsApp entre los adolescentes. En nuestro estudio, no solo el porcentaje de adolescentes que dicen no usar redes sociales es superior a los que mencionan que no acceden a WhatsApp, sino que, además, también están conectados diariamente en mayor medida a esta aplicación. Estos datos se corresponden con el estancamiento en el uso de redes sociales y el incremento del uso de mensajería instantánea entre la población general mayor de 18 años, tendencia que ha sido detectada por diferentes estudios en España (IAB, 2019; AIMC, 2020). El elevado porcentaje de adolescentes conectados diariamente a través de estos servicios se man- 
tiene en la línea de lo apuntado por Anderson y Jiang (2018) para los adolescentes estadounidenses; datos que dan cuenta del alto grado de interconexión social que mantienen los jóvenes en todo momento.

En cuanto al tipo de dispositivos que utilizan para acceder al mundo online, se puede hablar, sin ninguna duda, de una generación multipantalla en la que se manifiesta claramente la convergencia mediática. Los smartphones son los dispositivos más usados por los adolescentes, lo que favorece el uso intensivo de redes sociales y mensajería instantánea. También los ordenadores portátiles y las tabletas obtienen un elevado uso, con una tendencia a la sustitución de los segundos por los primeros cuando se eleva la edad. Por último, la propensión de los varones a usar «otros dispositivos» para conectarse nos da pistas de algunas diferencias en sus preferencias respecto a sus homónimas mujeres, constatando la tendencia hacia el juego interactivo realizado desde consolas (Ak et al., 2013) y que también se recoge en nuestro estudio.

Junto con las necesidades de socialización, la búsqueda de entretenimiento orienta el uso online de la adolescencia. Por un lado, destaca la cultura musical y, por otro, el consumo de contenidos (muchos de ellos probablemente divertidos) que les llegan en forma de recomendaciones y memes de las propias plataformas que, de esta forma, contribuyen a un empleo excesivo e incontrolado de las pantallas; aspecto que concuerda con la adhesión de la mayoría a la afirmación de que las redes sociales les hacen perder mucho tiempo. En la misma línea de esta preferencia lúdica, destaca el uso audiovisual de contenidos televisivos. Por el contrario, los menores no declaran acceder en gran medida a contenidos de riesgo, como páginas de apuestas deportivas o casino online, que informan sobre el consumo de drogas, de sexo para adultos o de búsqueda de pareja. Pero, simultáneamente, tampoco son muchos los que aprovechan otras oportunidades que les ofrece la red para informarse de la actualidad, sobre relaciones sexuales o comprar y vender online. Más concretamente, en lo que se refiere al uso de redes sociales, el tipo de uso realizado no difiere demasiado del que se hace del consumo de internet en general y se orienta a la socialización y el entretenimiento.

En relación con la valoración que hacen los adolescentes de las plataformas de redes sociales, encontramos visiones tanto positivas como negativas hacia estas. En este sentido, aunque consideran la pérdida de tiempo que les supone, los encuestados no piensan que las redes sociales les aíslen de la vida real. Son aquellos que están en los cursos más bajos los que muestran, en mayor proporción, su desacuerdo con esta idea. Cabe destacar un porcentaje que, sin ser muy alto, resulta preocupante en relación al llamado estrés del usuario (Mascheroni y Ólafsson, 2014), caracterizado con la presión que se siente de estar siempre disponible para contestar inmediatamente a las demandas de familiares y amigos en las redes sociales y WhatsApp. También merece ser mencionado el síndrome del FOMO (Fear of Missing Out), que se podría aplicar a un $22 \%$ de encuestados y sobre el que ya alertan los expertos (Bur- 
glass et al., 2017). Por último, la vulnerabilidad asociada al periodo de afianzamiento de la identidad en el que se encuentran los adolescentes se manifiesta en la presión que sienten algunos encuestados de lograr el reconocimiento social vía «likes» y «me gusta» en redes sociales. Para finalizar, los adolescentes eligen mayoritariamente Instagram para realizar la mayoría de sus actividades en redes sociales; y, a continuación, YouTube, que seleccionan para ver, especialmente, contenidos audiovisuales.

La limitación más clara de este trabajo está relacionada con la cobertura geográfica, puesto que está circunscrito a la Comunidad de Madrid. Por otra parte, su naturaleza descriptiva apunta a ulteriores análisis vinculados a la influencia de las diferentes variables sociodemográficas así como a la relación del uso aquí analizado con las motivaciones de los adolescentes. Por último, cabe destacar que este estudio ofrece tres aportaciones: por un lado, la confirmación de las tendencias en cuanto a las edades de conexión y acceso multipantalla, así como a la naturaleza de las actividades online. En segundo lugar, la detección de la relevancia de WhatsApp en comparación con el uso de las plataformas de redes sociales. Asimismo, es destacable la valoración los menores sobre las redes: creen que les hace perder tiempo, aunque no hasta el punto de aislarles de la vida offline.

Financiación: esta investigación se encuentran inmersa en el proyecto de $\mathrm{I}+\mathrm{D}+\mathrm{i}$ perteneciente al plan nacional «Redes sociales, adolescentes y jóvenes: convergencia de medios y cultura digital» (CSO2016-74980-C2-2-R).

\section{Referencias bibliográficas}

Aguaded, I. y Sánchez, J. (2013). El empoderamiento digital de niños y jóvenes a través de la producción audiovisual. AdComunica, 5, 175-196. doi: 10.6035/2174-0992.2013.5.11

AIMC (2020). Marco General de los Medios en España 2019. Recuperado de: https:// www.aimc.es/a1mc-c0nt3nt/uploads/2020/01/marco2020.pdf

Ak, S., Koruklu, N. y Yilmaz, Y. (2013). A study on Turkish adolescent's internet use: possible predictors of internet addiction. Cyberpsychology, Behavior, and Social Networking, 16(3), 205-209. doi: 10.1089/cyber.2012.0255

Anderson, M. y Jiang, J. (2018). Teens, social media and technology 2018. Report: Pew Research Center. Recuperado de: https://www.pewresearch.org/internet/ 2018/05/31/ teens-social-media-technology-2018/

Ballesta, F.J., Lozano, J., Cerezo, M.C. y Soriano, E. (2015). The internet, social networks and adolescence: A study base on secondary schools of the region of Murcia. Revista Fuentes, 16, 109-130. doi: 10.12795/revistafuentes.2015.i16.05

Burglass, S.L., Binder, J.F., Betts, L.R. y Underwood, J.D.M. (2017). Motivators of online vulnerability: The impact of social network site use and FOMO. Computers in Human Behavior, 66, 248-255. doi: 10.1016/j.chb.2016.09.055 
Cuesta, U. y Gaspar, S. (2013). Análisis motivacional del uso de smartphone entre jóvenes: una investigación cualitativa. Historia y Comunicación social, 18, 435-447. doi: 10.5209/ rev_HICS2013.v18.44252

Espín Álvarez, E. y Freire Muñoz, I. (2019). Relación entre el uso de internet para el entretenimiento y el aprendizaje escolar en estudiantes adolescentes del Ecuador. CienciAmérica: Revista de divulgación científica de la Universidad Tecnológica Indoamérica, 8(1), 138-157. doi: 10.33210/ca.v8i1.209

Fernández-Montalvo, J., Peñalva, A. e Irazabal I. (2015). Internet use habits and risk behaviours in preadolescence. Comunicar, 44(XXII), 113-120. doi: 10.3916/C44-2015-12

Fernández-Planells, A. y Figueras, M. (2012). Internet en las tareas escolares ¿obstáculo u oportunidad? El impacto de la red en los hábitos de estudio de alumnos de Secundaria de Barcelona y Lima. Sphera Publica, 12, 161-182. Recuperado de: http://www. redalyc.org/articulo.oa'id $=2972957701$

Fogg, B.J. (2008). Mass interpersonal persuasion: An early view of a new phenomenon. In Proceedings of the Third International Conference on Persuasive Technology, Persuasive 2008. Berlin: Springer. Recuperado de: http://captology.stanford.edu/ wpcontent/uploads/2014/03/MIP_Fogg_Stanford.pdf

García Jiménez, A. y Montes Vozmediano, M. (2020). Subject matter of videos for teens on YouTube. International Journal of Adolescence and Youth, 25(1), 63-78. doi: 10.1080/02673843.2019.1590850

García Jiménez, A., Tur-Viñes, V. y Pastor Ruiz, Y. (2018). Consumo mediático de adolescentes y jóvenes. Noticias, contenidos audiovisuales y medición de audiencias, Icono 14, 16(1), 22-46. doi: 10.7195/ri14.v16i1.1101

Garmendia, M., Garitaonandia, C., Martínez, G. y Casado, M.A. (2011). Riesgos y seguridad en internet: los menores españoles en el contexto europeo. Universidad del País Vasco, Bilbao: EU Kids Online. Recuperado de: https://www.observatoriodelainfancia.es/ ficherosoia/documentos/3155_d_Informe_EU_Kids_Online_Espa\%C3\%B1a_completo_red.pdf

Garmendia, M., Jiménez, E., Casado, M.A. y Mascheroni, G. (2016): Net Children Go Mobile: Riesgos y oportunidades en internet y el uso de dispositivos móviles entre menores españoles (2010-2015). Madrid: Red.es/UPV. Recuperado de: https://www.ehu.eus/ documents/1370232/0/Informe+NET+CHILDREN+RED.ES+España+2016. pdf/626251bc-f3f1-47ee-a06b-8401b91352df

Golpe-Ferreiro, S., Gómez-Salgado, P., Harris, S.K., Braña, T. y Rial, A. (2017). Diferencias de sexo en el uso de internet en adolescentes españoles. Behavioral Psychology, 25(1), 129-146. Recuperado de: https://www.researchgate.net/publication/317024119_ Diferencias_de_sexo_en_el_uso_de_internet_en_adolescentes_espanoles

IAB (2019). Estudio anual de redes sociales 2019. Recuperado de: https://iabspain.es/wpcontent/uploads/2019/06/estudio-anual-redes-sociales-iab-spain-2019_vreducida.pdf

INE (2019). Encuesta sobre equipamientos y uso de tecnologías de información y comunicación en los hogares. Madrid: INE. 
Jiménez Iglesias, E., Garmendia, M. y Casado del Río, M.Á. (2015). Percepción de los y las menores de la mediación parental respecto a los riesgos en internet. Revista Latina de Comunicación Social, 70, 49-68. Recuperado de: http://www.revistalatinacs.org/070/ paper/1034-UP/04es.html

Lenhart, A., Smith, A. y Anderson, M. (2015). Teens, social media and technology overview 2015: Smartphones facilitate shifts in communication landscape for teens. Washington, DC: Pew Research Center. Recuperado de: https://www.pewresearch.org/wp-content/ uploads/sites/9/2015/10/pi_2015-10-01_teens-technology-romance_final.pdf

Livingstone, S., Haddon, L., Görzig, A. y Ólafsson, K. (2011). Risks and safety on the internet: The perspective of European children. Full findings. London: LSE, EU Kids Online.

López-de-Ayala, M.C., Martínez-Pastor, E. y Catalina-García, B. (2019). «Nuevas estrategias de mediación parental en el uso de las redes sociales por adolescentes». El profesional de la información, 28(5), e280523.

Martínez-Pastor, E., Sendín-Gutiérrez, J.C. y García-Jiménez, A. (2013). Percepción de los riesgos en la red por los adolescentes en España: Usos problemáticos y formas de control. Análisi, 48, 111-130. Recuperado de: https://ddd.uab.cat/pub/analisi/analisi_ a2013m9n48/analisi_a2013m9n48p111.pdf

Mascheroni, G. y Ólafsson, K. (2014). Net children Go Mobile:risk and opportunities. Second Edition. Milano: Educatt. Recuperado de: https://www.researchgate.net/ publication/283320908_Net_Children_Go_Mobile_risks_and_opportunities_Second_ edition_Milano_Educatt

Masip, M. y Balagué, I. (2015). El uso del teléfono móvil en España. Barcelona: Instituto Psicológico Desconect@. Recuperado de: https://www.programadesconecta.com/wpcontent/uploads/2018/09/informe_moviles.compressed.pdf

Montes-Vozmediano, M.; García-Jiménez, A. y Menor-Sendra, J. (2018). Teen videos on YouTube: Features and digital vulnerabilities. Comunicar, 54(1), 61-69. doi: 10.3916/ C54-2018-06

Navarro, H., García, I., González, Z., Contreras, R. y Massana, E. (2012). El consumo multipantalla. Estudio sobre el uso de medios tradicionales y nuevos por parte de niños, jóvenes, adultos y personas mayores en cataluña. Quaders del CAC, 15(1), 91-100. Recuperado de: http://www.cac.cat/pfw_files/cma/recerca/quaderns_cac/q38_navarro_ et_al_es. pdf

Pastor Ruiz, Y., Martín Nieto, R. y Montes Vozmediano, M. (2019). Patrones de uso, control parental y acceso a la información de los adolescentes en la red. Estudios sobre el mensaje periodístico, 25(2), 995-1012. doi: 10.5209/esmp.64821

Pedrero-Esteban, L.M., Barrios-Rubio, A. y Medina-Ávila, V. (2019). Teenagers, smartphones and digital audio consumption in the age of Spotify. Comunicar, 60, 103-112. doi: 10.3916/C60-2019-10

Pérez-Torres, V., Pastor-Ruiz, Y. y Abarrou-Ben-Boubaker, S. (2018). YouTuber videos and the construction of adolescent identity. Comunicar, 55, 61-70. doi: 10.3916/C552018-06 
Reolid-Martínez, R., Flores-Copete, M., López-García, M., Alcantud-Lozano, P., Ayuso-Raya, M.C. y Escobar-Rabadán, F. (2016). Frecuencia y características de uso de internet en adolescentes españoles. Un estudio trasversal. Archivos Argentinos de Pediatría, 114(1), 6-13. Recuperado de: https://www.sap.org.ar/docs/publicaciones/ primero/2016/AO_Reolid_anticipo_15-12-15.pdf

Rial, A., Gómez, P., Braña, T. y Varela, J. (2014). Actitudes, percepciones y uso de internet y las redes sociales entre los adolescentes de la comunidad gallega (España). Anales de Psicología, 3(2), 642-655. doi: 10.6018/analesps.30.2.159111

Sendín, J.C., Gaona, M.C. y García, A. (2014). Nuevos medios: usos comunicativos de los adolescentes. Perspectivas desde los nativos digitales. Estudios sobre el Mensaje Periodístico, 20(1), 265-283. doi: 10.5209/rev_ESMP.2014.v20.n1.45231

Viñals, A. (2016). El ocio conectado, móvil, transmedia y multisoporte de los jóvenes en la era digital. Journal of Communication, 13, 99-113. doi: 10.14201/fjc20161399113 